\title{
No no-go: A remark on time machines ${ }^{\star}$
}

\author{
John Byron Manchak \\ manchak@uw.edu
}

\begin{abstract}
We present a counterexample to Krasnikov's (2002) much discussed time machine no-go result. In addition, we prove a positive statement: a time machine existence theorem under a modest "no holes" assumption.
\end{abstract}

Key words: Time Machines, Time Travel, Causality, General Relativity

\section{Introduction}

The following amounts to a minor remark. We simply claim that Krasnikov's (2002) time machine no-go result, as presented, cannot be true. In addition, we prove a positive statement: a time machine existence theorem under a modest "no holes" assumption.

\section{Background Structure}

We begin with a few preliminaries concerning the relevant background formalism of general relativity. ${ }^{1}$ An $n$-dimensional, relativistic spacetime (for $n \geq 2$ ) is a pair of mathematical objects $\left(M, g_{a b}\right) . M$ is a connected $n$-dimensional manifold (without boundary) that is smooth (infinitely differentiable). Here,

^ I thank Serguei Krasnikov, David Malament, and Christian Wüthrich for helpful comments on an earlier draft.

1 The reader is encouraged to consult Hawking \& Ellis (1973) and Wald (1984) for details. An outstanding (and less technical) survey of the global structure of spacetime is given by Geroch \& Horowitz (1979). 
$g_{a b}$ is a smooth, non-degenerate, pseudo-Riemannian metric of Lorentz signature $(+,-, \ldots,-)$ defined on $M$. Each point in the manifold $M$ represents an "event" in spacetime.

For each point $p \in M$, the metric assigns a cone structure to the tangent space $M_{p}$. Any tangent vector $\xi^{a}$ in $M_{p}$ will be timelike (if $g_{a b} \xi^{a} \xi^{b}>0$ ), null (if $g_{a b} \xi^{a} \xi^{b}=0$ ), or spacelike (if $g_{a b} \xi^{a} \xi^{b}<0$ ). Null vectors create the cone structure; timelike vectors are inside the cone while spacelike vectors are outside. A time orientable spacetime is one that has a continuous timelike vector field on $M$. A time orientable spacetime allows us to distinguish between the future and past lobes of the light cone. In what follows, it is assumed that spacetimes are time orientable.

For some interval $I \subseteq \mathbb{R}$, a smooth curve $\gamma: I \rightarrow M$ is timelike if the tangent vector $\xi^{a}$ at each point in $\gamma[I]$ is timelike. Similarly, a curve is null (respectively, spacelike) if its tangent vector at each point is null (respectively, spacelike). A curve is causal if its tangent vector at each point is either null or timelike. A causal curve is future-directed if its tangent vector at each point falls in or on the future lobe of the light cone.

For any two points $p, q \in M$, we write $p<<q$ if there exists a future-directed timelike curve from $p$ to $q$. We write $p<q$ if there exists a future-directed causal curve from $p$ to $q$. These relations allow us to define the timelike and causal pasts and futures of a point $p: I^{-}(p)=\{q: q<<p\}, I^{+}(p)=\{q$ : $p<<q\}, J^{-}(p)=\{q: q<p\}$, and $J^{+}(p)=\{q: p<q\}$. Naturally, for any set $S \subseteq M$, define $J^{+}[S]$ to be the set $\cup\left\{J^{+}(x): x \in S\right\}$ and so on. A chronology violating region $V \subseteq M$ is the set of points $p \in M$ such that there is a closed timelike curve through $p$. We say that a spacetime $\left(M, g_{a b}\right)$ is distinguishing if for all points $p, q \in M$, we have $I^{-}(p)=I^{-}(q) \Rightarrow p=q$ and $I^{+}(p)=I^{+}(q) \Rightarrow p=q$.

A point $p \in M$ is a future endpoint of a future-directed causal curve $\gamma: I \rightarrow M$ if, for every neighborhood $O$ of $p$, there exists a point $t_{0} \in I$ such that $\gamma(t) \in O$ for all $t>t_{0}$. A past endpoint is defined similarly. For any set $S \subseteq M$, we define the past domain of dependence of $S$ (written $D^{-}(S)$ ) to be the set of points $p \in M$ such that every causal curve with past endpoint $p$ and no future endpoint intersects $S$. The future domain of dependence of $S$ (written $D^{+}(S)$ ) is defined analogously. The entire domain of dependence of $S$ (written $D(S)$ ) is just the set $D^{-}(S) \cup D^{+}(S)$.

A set $S \subset M$ is achronal if no two points in $S$ can be connected by a timelike curve. A set $S \subset M$ is a slice if it is closed, achronal, and without edge. A spacetime $\left(M, g_{a b}\right)$ which contains a slice $S$ such that $D(S)=M$ is said to be globally hyperbolic. A set $S \subset M$ is a spacelike surface if $S$ is an $(n-1)$ dimensional submanifold (possibly with boundary) such that every curve in $S$ 
is spacelike. ${ }^{2}$

Finally, two spacetimes $\left(M, g_{a b}\right)$ and $\left(M^{\prime}, g_{a b}^{\prime}\right)$ are isometric if there is a diffeomorphism $\phi: M \rightarrow M^{\prime}$ such that $\phi_{*}\left(g_{a b}\right)=g_{a b}^{\prime}$. Two spacetimes $\left(M, g_{a b}\right)$ and $\left(M^{\prime}, g_{a b}^{\prime}\right)$ are locally isometric if, for each point $p \in M$, there is an open neighborhood $O$ of $p$ and an open subset $O^{\prime}$ of $M^{\prime}$ such that $O$ and $O^{\prime}$ are isometric, and, correspondingly, with the roles of $\left(M, g_{a b}\right)$ and $\left(M^{\prime}, g_{a b}^{\prime}\right)$ interchanged. We say a spacetime $\left(M^{\prime}, g_{a b}^{\prime}\right)$ is a (proper) extension of $\left(M, g_{a b}\right)$ if there is a proper subset $N$ of $M^{\prime}$ such that $\left(M, g_{a b}\right)$ and $\left(N, g_{a b \mid N}^{\prime}\right)$ are isometric. We say a spacetime is maximal if it has no proper extension.

\section{No No-Go}

In this section, we will first present Krasnikov's much discussed claim. ${ }^{3}$ Then, we will provide the counterexample. A few definitions will help us along. We begin by considering a distinction between local and non-local conditions on spacetime.

Definition. (Krasnikov) A condition $\mathfrak{C}$ is local if the following is true: For all spacetimes $\left(M, g_{a b}\right), \mathfrak{C}$ holds in $\left(M, g_{a b}\right)$ if and only if, for all open subsets $U$ of $M, \mathfrak{C}$ holds in the spacetime $\left(U, g_{a b \mid U}\right)$.

We note here that Krasnikov's definition of a local condition is strange; certain conditions (such as the non-existence of closed timelike curves) which are intuitively global, count as local. We will eventually exploit the unusual nature of this definition.

Definition. (Krasnikov) A spacetime is a $\mathfrak{C}$-spacetime if it satisfies local condition $\mathfrak{C}$. A $\mathfrak{C}$-spacetime $\left(M^{\prime}, g_{a b}^{\prime}\right)$ is a $\mathfrak{C}$-extension of a $\mathfrak{C}$-spacetime $\left(M, g_{a b}\right)$ if $\left(M^{\prime}, g_{a b}^{\prime}\right)$ is an extension of $\left(M, g_{a b}\right)$. A $\mathfrak{C}$-spacetime is $\mathfrak{C}$-extendible if it has a $\mathfrak{C}$-extension and is $\mathfrak{C}$-maximal otherwise.

Here is Krasnikov's central result which has been interpreted as the statement that "in classical general relativity a time machine cannot be built" (Krasnikov, 2002, p. 4109).

Claim. (Krasnikov) Any $\mathfrak{C}$-spacetime $\left(M, g_{a b}\right)$ has a $\mathfrak{C}$-maximal $\mathfrak{C}$-extension

2 Allowing $S$ to have a boundary is non-standard but the formulation introduces no difficulties. In particular, one may consider initial data on $S$ and determine its domain of dependence $D(S)$. See Hawking \& Ellis (1973, p. 201).

3 See Earman, Smeenk, \& Wüthrich (2009), Earman \& Wüthrich (2010), Manchak (2009a), Minguzzi (2009), Minguzzi \& Sánchez (2008), Monroe (2008), Olum \& Graham (2003), Smeenk \& Wüthrich (2010). 
$\left(M^{\prime}, g_{a b}^{\prime}\right)$ such that all closed causal curves in $\left(M^{\prime}, g_{a b}^{\prime}\right)$ (if any exist) are contained in the set $I^{-}[M]$.

Now we construct our counterexample. We begin by defining, in two steps, a certain spacetime property $\mathfrak{A}$. Then, we prove that it is local under Krasnikov's definition.

Definition. A spacetime $\left(M, g_{a b}\right)$ is almost maximal if it has a maximal extension $\left(M^{\prime}, g_{a b}^{\prime}\right)$ such that the set $M^{\prime}-M$ contains only a finite number of points.

Definition. A spacetime satisfies condition $\mathfrak{A}$ if it satisfies one of the following: (i) it is either maximal or almost maximal and contains closed timelike curves or (ii) it is neither maximal nor almost maximal.

Lemma. Condition $\mathfrak{A}$ is local.

Proof. Let $\left(M, g_{a b}\right)$ be an arbitrary spacetime satisfying $\mathfrak{A}$. We must first show that, for every open subset $U$ of $M$, the spacetime $\left(U, g_{a b \mid U}\right)$ satisfies $\mathfrak{A}$ as well. If $\left(M, g_{a b}\right)$ is neither maximal or almost maximal, then clearly every open subset $U$ of $M$ will be such that $\left(U, g_{a b \mid U}\right)$ is neither maximal nor almost maximal. So, every open subset $U$ of $M$ will be such that $\mathfrak{A}$ holds in $\left(U, g_{a b \mid U}\right)$. We turn to the other case: assume that $\left(M, g_{a b}\right)$ is either maximal or almost maximal. Since $\left(M, g_{a b}\right)$, by assumption, satisfies $\mathfrak{A}$, it follows that $\left(M, g_{a b}\right)$ must must have a chronology violating region $V \subset M$ (which is necessarily open). Let $U$ be an arbitrary open subset of $M$. If $\left(U, g_{a b \mid U}\right)$ is neither maximal nor almost maximal, then it satisfies $\mathfrak{A}$. If, on the other hand, $\left(U, g_{a b \mid U}\right)$ is either maximal or almost maximal, then clearly it is the spacetime $\left(M, g_{a b}\right)$ with some finite number of points removed. So $\left(U, g_{a b \mid U}\right)$ must have a chronology violating region $V^{\prime}$ where $V^{\prime}$ is just the set $V$ with a finite number points removed. So, $\left(U, g_{a b \mid U}\right)$ satisfies condition $\mathfrak{A}$.

Now, let $\left(M, g_{a b}\right)$ be an arbitrary spacetime which does not satisfy $\mathfrak{A}$. To complete the proof, we need only show that for some open subset $U$ of $M$, the spacetime $\left(U, g_{a b \mid U}\right)$ also fails to satisfy $\mathfrak{A}$. But this is easy. We know that $\left(M, g_{a b}\right)$ must be either maximal or almost maximal and free of closed timelike curves. Let $U$ be the set $M$ with one point removed. Clearly, the spacetime $\left(U, g_{a b \mid U}\right)$ is almost maximal. But it is also free of closed timelike curves. So $\left(U, g_{a b \mid U}\right)$ fails to satisfy $\mathfrak{A}$.

Now, in order to streamline the proof of the proposition below, we give the following lemma.

Lemma. An $\mathfrak{A}$-maximal spacetime contains closed timelike curves.

Proof. Let $\left(M, g_{a b}\right)$ be an $\mathfrak{A}$-maximal spacetime. Assume that $\left(M, g_{a b}\right)$ is free 
of closed timelike curves. We show a contradiction. We know that, because $\left(M, g_{a b}\right)$ is free of closed timelike curves and satisfies $\mathfrak{A}$, it must be neither maximal nor almost maximal. Let $\left(M^{\prime}, g_{a b}^{\prime}\right)$ be any maximal extension of $\left(M, g_{a b}\right)$. Note that this implies that the set $M^{\prime}-M$ contains an infinite number of points. In $\left(M^{\prime}, g_{a b}^{\prime}\right)$, let $p$ be any point in the boundary of $M^{\prime}-M$. And let $N$ be a neighborhood of $p$ which is small enough to permit the existence of a subset of $M^{\prime}-M$, disjoint from $N$, which contains an infinite number of points. Consider the spacetime $\left(M \cup N, g_{a b \mid M \cup N}^{\prime}\right)$. Clearly, it is an extension of $\left(M, g_{a b}\right)$. But since it is neither maximal nor almost maximal by construction, $\left(M \cup N, g_{a b \mid M \cup N}^{\prime}\right)$ is also an $\mathfrak{A}$-spacetime. So, $\left(M, g_{a b}\right)$ is $\mathfrak{A}$-extendible and therefore not $\mathfrak{A}$-maximal: a contradiction.

Finally, we are ready to present the counterexample.

Proposition. Krasnikov's claim is false.

Proof. Assume Krasnikov's claim is true. We show a contradiction. Let $\left(M^{\prime \prime}, g_{a b}^{\prime \prime}\right)$ be Misner spacetime. So, $M^{\prime \prime}=\mathbb{R} \times \mathbb{S}$ and $g_{a b}^{\prime \prime}=2 \nabla_{(a} t \nabla_{b)} \varphi+t \nabla_{a} \varphi \nabla_{b} \varphi$ where the points $(t, \varphi)$ are identified with the points $(t, \varphi+2 \pi n)$ for all integers $n$. Let $M$ be the set $\left\{(t, \varphi) \in M^{\prime \prime}: t<0\right\}$ and let $g_{a b}=g_{a b \mid M}^{\prime \prime}$.

Consider the spacetime $\left(M, g_{a b}\right)$. By construction, it is neither maximal nor almost maximal. So, it is an $\mathfrak{A}$-spacetime. Let $\left(M^{\prime}, g_{a b}^{\prime}\right)$ be any $\mathfrak{A}$-maximal, $\mathfrak{A}$ extension to $\left(M, g_{a b}\right)$. By the lemma above, $\left(M^{\prime}, g_{a b}^{\prime}\right)$ contains closed timelike curves. By Krasnikov's claim, these closed timelike curves must be contained in the set $I^{-}[M]$. But this leads to a contradiction: in any extension of $\left(M, g_{a b}\right)$, including $\left(M^{\prime}, g_{a b}^{\prime}\right)$, one can verify that $I^{-}[M]=M$ and that $M$ contains no closed timelike curves.

\section{Positive Result}

As mentioned by Earman \& Wüthrich (2010), it may be that something like Krasnikov's claim can be proven if it is sufficiently altered. ${ }^{4}$ But in this section, we wish to put aside worries concerning a possible no-go result and prove, under a modest "no holes" assumption, the existence of a time machine.

One condition which is sometimes imposed on spacetime is hole-freeness (Geroch, 1977). Formally, we say a spacetime $\left(M, g_{a b}\right)$ is hole-free if, for any spacelike surface $\Sigma$ in $M$ there is no isometric embedding $\theta: D(\Sigma) \rightarrow M^{\prime}$ into another spacetime $\left(M^{\prime}, g_{a b}^{\prime}\right)$ such that $\theta(D(\Sigma)) \neq D(\theta(\Sigma))$.

$\overline{4}$ Perhaps one can use the definition of a local condition given in Manchak (2009c) to prove a no-go theorem. 
It turns out that under hole-freeness, a time machine existence theorem can be proven (Manchak, 2009a). However, it has recently been shown by Manchak (2009b) and Krasnikov (2009) that hole-freeness may not be a physically reasonable condition. Indeed, some maximal, globally hyperbolic models (including Minkowski spacetime) are not hole-free.

Fortunately, another more reasonable "no holes" assumption can also be used to prove a time machine existence theorem. Recall that a causally simple spacetime $\left(M, g_{a b}\right)$ is one which is (a) distinguishing and (b) such that for all $p \in M, J^{+}(p)$ and $J^{-}(p)$ are closed (Hawking \& Sachs, 1974). Here, condition (a) concerns the causal structure of spacetime while condition (b) concerns "the limitations on the kind of gaps [holes] spacetime can have" (Hawking \& Sachs, 1974, p. 295).

Of course, one cannot use causal simplicity to prove a time machine existence theorem; no distinguishing spacetime can have closed timelike curves. But, it turns out that we can use condition (b) alone to find a result. And not only is this condition satisfied by all globally hyperbolic models (including Minkowski spacetime) but it is also satisfied by many acausal models as well (e.g. Gödel spacetime, Taub-NUT spacetime). In this sense, then, it is a more appropriate condition than hole-freeness. We have the following. ${ }^{5}$

Definition. A spacetime $\left(M, g_{a b}\right)$ is a time machine if (i) there is a spacelike slice $S \subset M$, a set $T \subset M$ with compact closure, and a chronology violating region $V \subset M$ such that $T \subset D^{+}(S)$ and $V \subset J^{+}[T]$ and (ii) every maximal extension of $D(S)$ which satisfies condition (b) contains some chronology violating region $V^{\prime}$.

Proposition. There exists a time machine.

Proof. Let $\left(M, g_{a b}\right)$ be Misner spacetime. So, $M=\mathbb{R} \times \mathbb{S}$ and $g_{a b}=2 \nabla_{(a} t \nabla_{b)} \varphi+$ $t \nabla_{a} \varphi \nabla_{b} \varphi$ where the points $(t, \varphi)$ are identified with the points $(t, \varphi+2 \pi n)$ for all integers $n$.

Let $S$ be the spacelike slice $\{(t, \varphi) \in M: t=-1\}$. It can be easily verified that, $D^{+}(S)=\{(t, \varphi) \in M:-1 \leq t<0\}$. Let $T$ be the compact set $\{(t, \varphi) \in$ $M: t=-1 / 2\}$. So, $T \subset D^{+}(S)$. Note that the set $\{(t, \varphi) \in M: t>0\}$ is a chronology violating region. Call it $V$. Clearly, $V \subset J^{+}[T]$. Thus, we have satisfied condition (i) of the definition of a time machine.

Now, let $\left(M^{\prime}, g_{a b}^{\prime}\right)$ be a maximal extension of $D(S)=\{(t, \varphi) \in M: t<0\}$ which satisfies condition (b). We show that it must contain closed timelike curves. Now, for every $k \in[0,2 \pi]$, let $\gamma_{k}$ be the null geodesic curve whose image is the set $\{(t, \varphi) \in M: \varphi=k \&-1<t<0\}$. Now, for each $k, \gamma_{k}$

$\overline{5}$ The reader is encouraged to compare this result with the one in Manchak (2009a). 
either has a future endpoint $p_{k}$ or not. Let $K$ be the set of all the endpoints $p_{k}$. Because $\left(M^{\prime}, g_{a b}^{\prime}\right)$ is maximal and satisfies condition (b), we know that $K$ must be a closed null curve. (Assume not. Because $\left(M^{\prime}, g_{a b}^{\prime}\right)$ is maximal, there will certainly be some point $p$ in $K$. But because $K$ is not a closed null curve, there will be some other point $q$ in the neighborhood of $p$ which is also in $K$ such that $q \notin J^{-}(p)$. But of course, $q$ is in the closure of $J^{-}(p)$ since $q \in K$. So $J^{-}(p)$ is not closed - a contradiction.)

Now, we can extend the coordinate system used in Misner spacetime to a neighborhood $K^{\prime} \subset M^{\prime}$ of $K$. Under this coordinate system, we have $K=$ $\left\{(t, \varphi) \in K^{\prime}: t=0\right\}$. Consider any point $p_{k} \in K$ and a neighborhood $U_{k} \subset K^{\prime}$ of $p_{k}$. Let $f_{k}: U_{k} \rightarrow \mathbb{R}$ be the function defined by $f_{k}(t, \varphi)=g_{a b}^{\prime}(t, \varphi)\left(\frac{\partial}{\partial \varphi}\right)^{a}\left(\frac{\partial}{\partial \varphi}\right)^{b}$. Of course, when the domain of $f_{k}$ is restricted to the set of points $(t, \varphi) \in U_{k}$ where $t \leq 0$, then $f_{k}(t, \varphi)=t$. The smoothness of $g_{a b}^{\prime}$ ensures the boundary conditions $f_{k}(0, \varphi)=0$ and $\frac{\partial}{\partial t} f_{k}(0, \varphi)=1$ are satisfied. Clearly then, there must be an $\epsilon_{k}$ such that $f_{k}(t, \varphi)>0$ for all $t \in\left(0, \epsilon_{k}\right]$. Now, let $\epsilon: K \rightarrow \mathbb{R}$ be the function defined by $\epsilon\left(p_{k}\right)=\epsilon_{k}$. Note that the smoothness of $g_{a b}^{\prime}$ allows us to choose our $\epsilon_{k}$ so that $\epsilon$ is a continuous function. Because $K$ is compact, $\epsilon$ takes on a minimum value (call it $\left.\epsilon_{\min }\right) .{ }^{6}$ Next, let $V^{\prime}$ be the set $\{(t, \varphi)$ : $\left.0<t \leq \epsilon_{\min }\right\}$. Clearly, on $V^{\prime}$, we have $g_{a b}^{\prime}\left(\frac{\partial}{\partial \varphi}\right)^{a}\left(\frac{\partial}{\partial \varphi}\right)^{b}>0$. Now, let $w$ be any point in $V^{\prime}$ and consider the curve $\gamma: I \rightarrow V^{\prime}$ through $w$ with tangent vector

$\xi^{a}=\left(\frac{\partial}{\partial \varphi}\right)^{a}$ at every point. Because $\gamma$ is contained entirely within $V^{\prime}$, we know that $g_{a b}^{\prime} \xi^{a} \xi^{b}>0$. Thus, $\gamma$ is a closed timelike curve and we have satisfied condition (ii) of the definition of a time machine.

\section{References}

Earman, J., Smeenk, C., \& Wüthrich, C. (2009). Do the laws of physics forbid the operation of time machines? Synthese, 169, 91-124.

Earman, J., \& Wüthrich, C. (2010). Time machines. In E. N. Zalta (ed.), Stanford Encyclopedia of Philosophy, http://plato.stanford.edu/entries/timemachine/.

Geroch, R. (1977). Prediction in general relativity. In J. Earman, C. Glymour, \& J. Stachel (Eds.), Foundations of space-time theories, Minnesota studies in the philosophy of science Vol. VIII (pp. 81-93). Minneapolis: University of Minnesota Press.

Geroch, R., \& Horowitz, G. (1979). Global structure of spacetimes. In S. Hawking \& W. Isreal (Eds.), General relativity: An Einstein centenary survey (pp. 212-293). Cambridge: Cambridge University Press.

Hawking, S., \& Ellis, G. F. R. (1973). The large scale structure of space-time. Cambridge: Cambridge University Press.

$\overline{6}$ See Wald (1984, p. 425). 
Hawking, S., \& Sachs, R. (1974). Causally continuous spacetimes. Communications in Mathematical Physics, 35, 287-296.

Krasnikov, S. (2002). No time machines in classical general relativity. Classical and Quantum Gravity, 19, 4109-4129.

Krasnikov, S. (2009). Even the Minkowski space is holed. Physical Review D 79, 124041.

Manchak, J. (2009a). On the existence of 'time machines' in general relativity. Philosophy of Science, 76, 1020-1026.

Manchak, J. (2009b). Can we know the global structure of spacetime? Studies in History and Philosophy of Modern Physics, 40, 53-56.

Manchak, J. (2009c). Is spacetime hole-free? General Relativity and Gravitation, 41, 1639-1643.

Minguzzi, E. (2009). Chronological spacetimes without lightline lines are stably causal. Communications in Mathematical Physics, 288, 801-819.

Minguzzi, E., \& Sánchez, M. (2008). The causal hierarchy of spacetimes. In Alekseevsky, D. \& Baum, H. (Eds.), Recent developments in pseudoRiemannian geometry (pp. 299-358). Zürich: European Mathematical Society.

Monroe, H. (2008). Are Causality Violations Undesirable? Foundations of Physics, 38, 1065-1069.

Olum, K., \& Graham, N. (2003). Static negative energies near a domain wall. Physics Letters B, 554, 175-179.

Smeenk, C., \& Wüthrich, C. (2010) Time travel and time machines. In C. Callender (Ed.), The Oxford handbook of time, Oxford: Oxford University Press.

Wald, R. (1984). General relativity. Chicago: University of Chicago Press. 